\section{Response to Veenstra et al.}

We thank Veenstra et al. ${ }^{1}$ for the letter and interest in our study, ${ }^{2}$ which forecast the combined impact of cancer population testing and reproductive carrier screening in all Australian adults aged 18-25 years (2.6 million individuals).

Our results demonstrated preventive benefits and costeffectiveness in offering genomic screening to young adults in a single health-care system. Benefits were represented in disability-adjusted life years (DALYs) and incremental costeffectiveness ratio (ICER). Our findings will help inform the discussion regarding population-based genomic testingrightly described by Veenstra et al. as "one of the aspirational goals of precision medicine." We take our calculations and conclusions seriously, and make the following points:

1. Beyond preventing cancer deaths, we also calculated the benefits of preventing and delaying onset of nonfatal cancers (breast and colorectal). Veenstra et al. describe how they divided the total number of DALYs per cancer type presented in Supplemental Table 1 of our study by the number of cancer deaths prevented per cancer type, to calculate the number of DALYs per death prevented. They then compared this figure with other studies, including their own. However, our DALY calculations also included prevention of nonfatal cancers (not just cancer deaths).

Given most breast and colorectal cancers are now nonfatal, we considered including the benefits of preventing nonfatal cancers was important. We represented these benefits by calculating increases in life expectancy and life quality gained through early detection for those at high risk.

For example, an 18-25-year-old man with no known cancer family history, in whom a pathogenic $M L H 1$ variant is detected by population screening, may be otherwise unaware of his risk. After detection, he commences intensive surveillance for colorectal cancer prevention from age 25 to 60 following Australian standard of care, ${ }^{3}$ involving chemoprevention (aspirin) and annual colonoscopy. Adenomatous polyps may be detected early and removed, rather than developing into cancer, as expected. Yet colorectal cancer may eventually develop later in life (say age 70). This cannot be considered a "death prevented" in our model, but we wanted to include the DALYs gained from these unaccounted-for years of extended life quality and expectancy as a result of early risk detection.

As expected, this caused an increase in the number of DALYs gained for breast and colorectal cancer. We found preventing or delaying the onset of nonfatal colorectal cancers accounted for the majority of colorectal cancer DALYs gained $(>60 \%)$-more than preventing deaths. This effect was observed to a lesser extent in breast cancer, where prevention or delayed onset of cancer due to nonsurgical interventions is lower. ${ }^{4}$ We calculated DALYs gained and increases in life expectancy due to delayed onset of cancer using mortality and survival data for individuals in high-risk breast and colorectal cancer surveillance, compared with individuals not in surveillance. ${ }^{5-8}$

2. DALY calculations for cancer prevention in our study were moderately inflated due to an unrelated calculation issue. Prompted by the letter, we rechecked the entire model code. We identified a coding issue that resulted in DALYs being inadvertently counted twice in certain parts of the cancer model -once in extended life expectancy calculations, and again in improved life quality calculations. This inflated cancer-related DALYs, not DALYs prevented due to reproductive screening. No other issues were identified during the thorough code check. We subsequently removed the double-counted DALYs, and the ICER of combined screening for all seven conditions (AUD\$400 per test) increased from $\$ 4038$ ( $\$ 4$ to $\$ 7740$ ) to $\$ 7286$ ( $\$ 239$ to $\$ 14,034)$. Critically, this did not affect the overall conclusion of our study, i.e., that combined genomic screening for multiple conditions is highly cost-effective, well below the threshold of AUD\$50,000/DALY prevented.

Further, at AUD $\$ 200$ per test, even after adjustment, screening still approached cost-saving for the health system per our original conclusion (ICER $=$ AUD\$80/DALY). We have included a modified version of Supplemental Table 1 as supplementary material to this letter with adjusted figures. The adjustment changed the conclusion for cost-effectiveness for ovarian cancer screening alone, and combined colorectal and endometrial cancer screening, which were marginally cost-effective in the previous analysis $(\$ 39,350$ and $\$ 44,939$ / DALY respectively). These rose above the cost-effectiveness threshold (\$84,837 and \$93,193/DALY respectively). No other ICER calculations crossed the $\$ 50,000 /$ DALY threshold after adjustment.

Using adjusted figures, we replicated Veenstra et al.'s calculations, for comparison with other studies and their own work. Based on revised calculations, we calculated that population genomic screening would result in prevention of 166 colorectal cancer cases (attributable to MLH1/MSH2 variants), for 2183 DALYs gained (13.2 DALYs per case prevented). We then forecast an additional 295 individuals with $M L H 1 / M S H 2$ variants would develop cancers, but have life expectancy extended by access to chemoprevention and intensive screening. For this group, we calculated 3473 DALYs gained (11.8 DALYs per case). The difference between the groups was modest, because life expectancy for high-risk individuals in intensive colorectal cancer screening is reported to be close to population average (5-year survival after colorectal cancer diagnosis in intensive screening is 0.94 [ref. $\left.{ }^{8}\right]$ ). A one-way sensitivity analysis on this 5 -year survival 
rate (reducing survival from 0.94 to 0.70 , a more conservative estimate) only negligibly affected the overall ICER of combined screening, increasing from AUD\$7286 (\$239 to $\$ 14,034$ ) to AUD\$7445 (\$284 to $\$ 14,671$ ).

For breast cancer, we forecast 1278 breast cancer cases prevented (attributable to BRCA1/BRCA2 variants), resulting in 9148 DALYs gained (7.2 DALYs per case prevented). This is more comparable with findings from previous studies, and Veenstra et al.'s work. In addition, we forecast there would be 1918 women with $B R C A 1 / B R C A 2$ variants who still developed breast cancer, but had life expectancy extended due to intensive screening (annual mammogram and magnetic resonance imaging $[\mathrm{MRI}])$. We estimated intensive screening would improve 15 -year breast cancer survival by $21.6 \%$ (ref. $^{5}$ ) from 0.6274 (ref. $^{6}$ ) to 0.7629 , resulting in 7173 DALYs gained (3.8 DALYs per case).

In summary, after diligently rechecking our model, we identified a double-counting issue that had moderately inflated our DALY calculations, but did not impact study conclusions. The remaining difference in DALYs can be explained by the fact that nonfatal cancers prevented, and the delayed onset of cancer, are included in our model.

We thank Veenstra et al. again for their correspondence and interest in our study.

\section{SUPPLEMENTARY INFORMATION}

The online version of this article (https://doi.org/10.1038/s41436019-0581-3) contains supplementary material, which is available to authorized users.

\section{DISCLOSURE}

The authors declare no conflicts of interest.

Publisher's note: Springer Nature remains neutral with regard to jurisdictional claims in published maps and institutional affiliations.
Paul Lacaze, PhD (D ${ }^{1}$, Jane Tiller, MGenCouns ${ }^{1}$, Yining Bao, BSc $c^{1,2}$, Moeen Riaz, $P h D^{1}$, Ingrid Winship, $M D^{3}$ and Lei Zhang, $\mathrm{PhD}^{1,4}$

${ }^{1}$ Department of Epidemiology and Preventive Medicine, School of Public Health and Preventive Medicine, Monash University, Melbourne, VIC, Australia; ${ }^{2}$ School of Public Health, Nantong University, Nantong, Jiangsu, China; ${ }^{3}$ Department of Genomic Medicine, Royal Melbourne Hospital; Department of Medicine, Royal Melbourne Hospital, University of Melbourne, Melbourne, VIC, Australia; ${ }^{4}$ Department of Epidemiology and Biostatistics, School of Public Health, Xi'an Jiaotong University Health Science Center, Xi'an, Shaanxi, China. Correspondence: Paul Lacaze (paul. lacaze@monash.edu)

\section{REFERENCES}

1. Veenstra DL, Guzauskas G, Peterson J. Cost effectiveness of population genomic screening. Genet Med. 2019. https://doi.org/10.1038/s41436019-0580-4 [Epub ahead of print].

2. Zhang $\mathrm{L}$, Bao $\mathrm{Y}$, Riaz $\mathrm{M}$, et al. Population genomic screening of all young adults in a health-care system: a cost-effectiveness analysis. Genet Med. 2019 Feb 18; https://doi.org/10.1038/s41436-019-0457-6 [Epub ahead of print].

3. NSW Government Cancer Institute. EviQ Cancer Treatment Guidelines 619 v4. Genetic testing for hereditary mutations in the mismatch repair genes (MMR-genes). Government report/guidelines. https://mww.eviq.org.au/ cancer-genetics/genetic-testing-for-heritable-mutations/619-genetic-testingfor-heritable-mutations-in-the (2018). Accessed 14 June 2019.

4. Kuchenbaecker KB, Hopper JL, Barnes DR, et al. Risks of breast, ovarian, and contralateral breast cancer for BRCA1 and BRCA2 mutation carriers. JAMA. 2017;317:2402-2416.

5. Evans DG, Harkness EF, Howell $A$, et al. Intensive breast screening in BRCA2 mutation carriers is associated with reduced breast cancer specific and all cause mortality. Hered Cancer Clin Pract. 2016;14:8.

6. Schmidt MK, van den Broek AJ, Tollenaar RA, et al. Breast cancer survival of BRCA1/BRCA2 mutation carriers in a hospital-based cohort of young women. J Natl Cancer Inst. 2017;109:djw329.

7. Australian Government, Australian Institute of Health and Welfare. Cancer in Australia 2017 Report. Cat. no. CAN 100. https://www.aihw.gov.au/ getmedia/3da1f3c2-30f0-4475-8aed-1f19f8e16d48/20066-cancer-2017. pdf.aspx?inline=true (2017). Accessed 14 June 2019.

8. Moller P, Seppala T, Bernstein I, et al. Cancer incidence and survival in Lynch syndrome patients receiving colonoscopic and gynaecological surveillance: first report from the prospective Lynch syndrome database. Gut. 2017;66:464-472.

Advance online publication 15 July 2019. doi:10.1038/s41436-019-0581-3 